\title{
Bibliographie des traditions et de la littérature populaire du Poitou.
}

L'ancienne province du Poitou, qui parle un dialecte de langue d'oïl, se compose des trois départements actuels de la Vienne, des Deux-Sèvres et de la Vendée. Bien que ce pays, sauf pour les chansons, n'ait pas été l'objet d'une exploration aussi complète au point de vue du folk-Lore proprement dit que la Bretagne, et quelques autres provinces de France, on y trouvera pourtant l'indication de numbreux ouvrages uì se rencontrent, en quantités variables, des renseignements relatifs aux traditions, aux mours et aux usages.

Nous avons suivi à peu de chose près le mème plan que dans nos deux précédentes publications ${ }^{1}$ sur le même sujet; après avoir relevé les glossaires et les uuvrages de linguistique, si souvent intimement liés à la littérature orale, nous avons ensuite passé en revue les traditions, moeurs et usages - les contes populaires - les Chansons - les Nö̈ls - les Devinettes - les Formulettes et les prières populaires - les Proverbes - les Costumes - les livres populaires et les almanachs - les faïences, l'imagerie et la plomberie - le théâtre populaire.

Plusieurs savants du Poitou, et parmi eux M. Léo Desaivre, ont bien voulu nous envoyer de précieuses notes qui ont singulièrement enrichi notre travail, et sans les quelles nous serions loin de de présenter un ensemble aussi complet. Qu'il accepte nos remerciements et ceux de nos lecteurs pour son dévoué concours.

\section{Aire linguistique du patois poitevin.}

Drkux Du Radiek. Bibliothèque histor. et crit. du Poitou. 5 vol. Paris, Ganeau, 1754. Journal de Verdun fevr. 1758, t. 93, p. 123.

Dupin, préfet des 2. Sèvres. Statistique des 2. S. I ter mém. p. $56 ; 20$ mém. p. 204 et 205; mém. de la soc des antiq. de Fr. tome Ier p. I95 et seqq DE La Fontenelle iJE Vaudoré. Recherches sur la langue poitevine. Bull. de la Société d'agriculture etc. du Poitou 1830.

Communications de M. M. Cardin (de Poitiers) et De la Fontenelle de Vaudoré au Congrès scientifique de Blois 1836 .

1 Bibliographie des traditions et de la littérature populaire de la Bretagne (Revue Celtique t. V p. 277-339). Bibliographie des traditions et de la littérature populaire de l'Alsace. Strasbourg, Noiriel, 1883 (tirage d̀ part du Polybiblion de Novembre 1882). 
Schnakenbukg. Tableau synoptique et comparatif des idiomes populaires ou patois de la France. Berlin, 1840 .

FAvre. Glossaire du Poitou. Introduction p. 2.

Tourtoulon (Ch. de) et Bringlikr (O.). Rapport sur la limite géographique de la langue d'oc et de la langue d'oïl. Archives des Missions $3^{\text {e }}$ sér. t. III, 18;6. Il existe un tirage à part.

Sur la limite du Poitevin of. la page 54.

Boucherie (A.). Patois de la Saintonge; curiosités étymologiques et grammaticales. Angoulème, A. Nadaud, 1865.

Dans l'introduction M. Boucherie rattache le Poitevin et le Saintongeais à la languce d'oïl.

$$
\text { Patois. Glossaires et Grammaires. }
$$

Drouher (Jean). La Moirie de Sen Moixont o les vervedé de tretoute lez autre (la Mairie de St. Maixeut où il est parlé de toutes les autres).

La Mizaille à Tauni, comédie en vers (sic) avec l'explication des mots poitevins les plus difficiles à savoir. Poitiers, Pierre Amassard, 1660 et I66I.

On peut considérer cettc explication comme le premier essai de Glossaire fait en Poitou.

Alfred Richard, Archiviste de la Vienne. Les ceuvres de Jean Drouhet, Mo apothicaire à St. Maixeut. (I a moirie de Sen Moixont - la mizaille à Tauni - Dialogue Poictevin de Michea, Pérot, Jouset Huguenots et Lacas Catholique sur ce qui s'est passé à la conversion de Mr Cotibi, ministre de Poictiers en 1660 - Lez bon et bea prepou do bounhoume Bretau - La défonse dos enfous de la ville de Sen Moixont contre les railleries do gens de Poetey - le grou fremage d'Hollande.) Avec notes expliquant les mots difficiles. Poitiers, Druineau, 1878.

La Revelliére-Lépeaux (L. M.). Notice du Patois Vendéen. Mém. de l'Acad. Celt. t. III (1809) p. 267-290. Il existe un tirage à part sans pagination spéciale.

- Notice du patois vendéen, précédé d'une biographie de l'auteur. Niort, 1868, gr. in 80 de $80 \mathrm{p}$.

- Essai d'un vocabulaire vendéen. Mém. de l'Acad. Celtique t. III (1809) p. $384-398$.

De i.A Fontenki.le de Vauloré. Recherches sur la langue poitevine. Poitiers, F. A. Saurin, in $8^{0}$. s. d. (P. G.)

Les exemplaires complets comprennent une seconde purtie pour la.grammaire etc.

Pofir I'Avavr. De l'Influence du langage poitevin sur le style de Rabelais. Paris, 1855, in 80. (Extr. de la Revue des provinces de l'Ouest. Nantes, A. Gueraud.)

Ducisst-Matifntix. Etude sur le patois poitevin. Kevue des provinces de l'Ouest. Nantes, A. Guéraud, 1858.

AUvé (Léon). Du langage populaire en Vendée. Napoléon-Vendće, 1858.

Pressac (biblre de la ville de Poitiers). Glossaire à la suite des poésies patoises de l'abbé Gusteau. Poitiers, Henri Oudin, 1855, in 12. 61.

(Rondier.) Quelques mots du patois poitevin. (le Mellois 28 Juillet 186r.)

- Génie du patois poitevin par un paysan. (le Mellois 14 Juillet, 21 Aoút, I er Sept. 1861 .)

,Gennes (Ch. de). Sur l'œuvre du patois Poitevin. Poitiers, Dupré, 1863, broch. in $8^{\circ}$.

Beauchet-Filleau. Essai sur le patois poitevin. Melle, 1863, in 80 . C'est un glossaire avec grammaire.

DReuX du RAlirer. Essai sur le langage poitevin. Niort, 1866, in $8^{\circ}$.

Digast-Matireux (Ch.). Réimpression de l'article du Journal de Verdun: Lettres sur l'orig. des langues espagnole et italienne ou essai sur la langage poitevin de Dreux du Radier, voir cidessus.

Duval (L.). Etudes critiques sur le patois poitevin. Niort, Ch. Mercier, $186 \%$, in $8^{\circ}$. 
LEVRIER (Gabriel). Dictionnaire étymologique du patois poitevin. Niort, Ch. Mercier, 1867 , in 80 de $195 \mathrm{p}$.

Favre (L.). Glossaire du Poitou, de la Saintonge et de l'Aunis, précédé d'une introduction sur l'origine, le caractère, les limites, la grammaire et la bibliographie des patois poitevin et saintongeais. Niort, 1868 , in $8^{\circ}$ de $35^{6} \mathrm{p}$.

LalaANNe (abbé). Glossaire du patois Poitevin. Poitiers, 1867 , in $\mathbf{8}^{\circ}$.

Forme le tome $X X X I I$ 2e partie des Mèm. de la Soc. des Ant. de l'Ouest.

Cf. not. de Paul Meyer Rev. Soc. savantes $4^{\theta}$ sér. t. IX p. 405.

Rousseau (abbé). Glossaire poitevin. Paris, 1869, in 80 de $95 \mathrm{p}$., a paru à la même époque dans la Rev. de l'Aunis, de la Saintonge et du Poitou. Boucherie. Le dialecte poitevin au XIIIe siècle. Paris, Pedone Lauriel, in 80,1873 .

Petit Glossaire poitevin - à la suite des Etudes sur les poësies de Christophe des Frances, seigueur de la Châlonnière et de la Jalousière, imprimées à Niort chez Th. Portau, 1595, et intitulé:

Histoire des poëtes compris au grand Olympe et ensuivant la Métamorphose d'Ovide.

Favre (L.). Supplément au dictionnaire du Poitou. Niort, L. Favre, 188I, gr. in $8^{0}$.

Traditions, Mceurs et Usages.

Bolchet (Guillaume). Les Serées. Poitiers, G. Bouchet, 1584 , in $4^{0}$.

Yver (Jaques, Sgr. de Plaisance). Le printemps d'hiver, conten. plus. hist. discourues en cinq journées, en une noble compagnie au chateau de Printemps. Niort, Thomas Portau, 1598 , pet. in 12 (pourrait être mis parmi les livres populaires anciens).

Discours facétieux des finesses de Croustelle, accommodé aux affaires de ce temps. Aux admirateurs de la tournure moderne, jouxte la copie, imprimé à Poitiers par Pierre Poyrier, imprimeur s. d. gloss. Favre.

Bodiv (Jean, né à Angers, mort à Laon en 1596). (Bibl. Poit. Clouzot 1878.) Le fléau des démons et sorciers. Niort, David du Terroir, 1616, in $8^{\circ}$.

Discours lamentables et espouvantables des merveilleux ténébres advenus

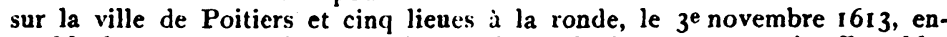
semble les estranges signes de feu en forme de dragon, avec cris effroyables et demeurèrent l'espace de trois jours sans y avoir aucune clarté. Lyon, Doret, 1614 , in 8:?

De la Haye: (Jean, baron des Coustaux). Les mémoires et recherches de France et de la Gaule aquitanique ì Paris. Guillon, 1619, pet. in 80 .

Collardeau (Julien). Feste de village par Julien Collardeau, procureur du Roy a Fontenay (1637). Fontenay, Robuchon, s. d., br. in $8^{\circ}$. (Publié par les soins de M. Dugast-Matifeux.)

Drouchv:l (Jean). I M Moirie de Sen Moixont 1660 , ouv. cité.

On $y^{\prime}$ trouve la description d'un festin de mairie et des détails de maurs.

Malchin (A.). Histoire de Saintonge, Poitou, Aunis et Angoumois. SaintJean-d'Angély, 167I, 2 parties en un vol., in folio.

DrEIX DC RADIER. Bibliothèque historique du Poitou. 1754. Ouvr. cité. Le t. I P. 368 et sqq. contient un long article sur les raretés du Poitox, extrait du Reductorium repertorium et Dictionnarium morale etc. de Petrus Berchorius Poitevin (mort en 1362) L. IV C. 43 éd.Cologne 1692. le passage est relatif a des superstitions sur les oiseaux; sur le privilìges qu'ont certaines familles de chasser les serpents etc.

A ubin. Histoire des diables de Loudun, ou la possession des religieuses Ursulines, et de la condannation et du supplice d'Urbain Grandier, curé de la même ville. Cruels effets de la vengeance du cardinal de Richelieu. A Amsterdam, 1752, in 12.

JomyneAd DEs LogEs, fondateur du affiches du Poituu ier Janv. 1773 31 Dec. 1781 - plus. art. dans cette précieuse collection.

Dumoustier DE I.A Fovr. Essai sur l'Hist. de Loudun, 2 vol. in 80; Poitiers, Chevrier, 1778 . 
Impatdeal. Abrégé de l'histoirc du Poitou, contenant ce qui s'est passé de plus remarquable dans cette province, depuis le règne de Clovis jusqu'au commencen ent de ce siècle. Poitiers, 1782, 6 vol. in 12. Errata de l'abregé de l'hist. du P. ou lettres à M. Thibeaudeau suivies d'un petit Commentaire par M. *** (Allard de la Resniere en France 1783) plus. fascicules dont le dernier en resté ms.

Thabacdfal: Histoire du Poitou, nouvelle édition précédée d'une introduction, par M. H. de Sainte-Hermine, avec notes. Niort, Robin, 1839, 3 vol. in 80 .

LA Val.Lée etc. Voyage dans les départements de la Fr. (Vienne, Deux-Sèvres,

Vée). Paris, Desenne etc., 1794, in $8^{\circ}$.

DORFEliLle. Dissertation sur l'existence des dragons, présentée à l'administration centrale du Département des Deux Sèvres à la séance du I 6 fructidor de l'an 6. par le citoven C. H. W. Dorfenille. A Saint Maixent chez F. Lainé. An VII in 12 , de XVI-59 pp.

Cet opuscule, des plus curieux par la crédulité de l'auteur fermement persuadé de l'existence des dragons, rentrerait dans les généralités, si en quelques passages et notamment p. 25 et 26, il ne parlait d'une légende poitevine vì un soldat combat le dragon de Niort avec un masque de verre, afin de se priserver du venin que lance le monstre.

Oraison funèbre du Mardigras, prononcé le 29 pluviose au IX sur la place de la Brèche de la ville de Niort etc. Niort de l'imprimerie de P. A. Elie au IX in 12.

JACQUiN (E.). Annuaire statistique du départemeut des Denx-Sèvres an XIII, (1804 et 1805). Niort, Plisson, an XIII, un vol. in $8^{\circ}$, avec la carte hydro. graphique du département.

VENDÉ (Annuaire statistique du département de la) pour 1803-1804, par le citoyen Cavoleau, se vend a Fontenay chez Goichot, in $8^{\circ}$.

Trioi.t.r. Antiquités et monuments du Poitou. 1804 in 80.

Dupis. Mémoires statistiques du département des Deux-Sèvres. Paris an XII. in $\mathbf{f}^{\circ}$.

Piet. Mémoires laissés à mon fils. Noirmoutiers 1806 , in $4^{0}$.

Joliskeal des Loriks. Sur les Noces noires des marais du Bas-Poitou. Mém. de l'Acad. Celt. t. V (18ro) p. 275-280.

BARRÉ DE JaIr.AIs. Essai sur les mours, l'administration et les besoins de la Vendée i815.

Journeat Des Loifis. Note sur le dragon de Poitiers et celui de Niort. Mém. de l'Acad celtique $1809 n^{0}{ }_{13}$.

Richer (Edouard). Statistique de Noirmoutiers, vers 1820 ?

DUPıN (Baron). Notice sur quelques fêtes et divertissements populaires du département des Deux-Sèvres. Mém. de la Société des Antiq. de France t. IV (1823) p. $104-127$.

Dufour. De l'ancien Poitou et de sa capitole. Poitiers, Loriot, 1826, in $8^{0}$. Souvenirs pittoresques du Poitou A. Noël. 1828 , in $f^{0}$.

MassÉ (Isidore). La Vendée poétique et pittoresque. Nantes, 1829, in $8^{\circ}$.

Marchés de louage et de fiançailles des garçons et des filles dans la Vendée. Magasin pittoresque 1834 p. 135.

Mérimée (Prosper). Notes d'un voyage dans l'Ouest de la France. Paris, Fournier, 1836 in 80.

La Fontenfile de Vaudorí. Notes sur l'nle Dieu, 1836 , in $8^{\circ}$.

Le même - Les arts et métiers à Poitiers, pendant les XIIIe, XIVo et XVe siècles. Poitiers, Saurin, 1837 , br. in $80^{\circ}$.

Dartigr. Le Poitou Pittoresque. $183^{8}$ in $4^{\circ}$.

Gueriniere. Histoire générale du Poitou I838-40, 2 vol. in $8^{\circ}$;

Notice sur les feux de la St. Jean dans le cant. de Gençay par' M. NicolasClémot. Soc. des antiq. de l'O., Bull. 1838 .

Savarr (chef de bataillon du génie). Notiçe sur les huttiers de la Sèvre. Mémoires de la Soc. de statistique des Deux Sèvres. $1838-1839$. 
Guerry. Note sur les usages et traditions du Poitou. Mém. Soc. Ant. de France VIII 1839, p. 451-465.

Feux de joie a St fean -- Mariage - rubans de la mariée - baufs bénis jour de St. Blanc - ruf coquatri - chanson de la mariée.

Bellin de t.A Liborliǩre. Second rapport sur des gâteaux d'une forme par. ticulière. Poitiers, Saurin, $184^{\circ}$, br. in $8^{\circ}$.

- Le ter mémoire a pour titre: Sur Mélusine et des gâteaux qui la représentent par M. de la Liborlière. Bull, de la soc. des antiq. de l'O., I840, avec fig.

La fontenelle de Vaudork. Chronique fontenaisiennes 1841, in 80.

De la Vili.eglilie (A.). Mours et Coutumes du Poitou. Bull. Soc. des Ant. de l'Ouest $4^{\mathrm{e}}$ Trimestre 1842, p. 300--312. Avec la chanson de la Mariée en patois.

Richarn (J., avocat). Notice sur l'établissement des rosières de La mothe St. Heraye et son fondateur. Soc. de stat. des $2 \mathrm{~S}$. Livraison 1843-44.

De Lastic st. Jal. Zoologie du départ. des 2 Sèv. Soc. de stat. des $2 \mathrm{~S}$. Livraison $1843-44$.

Mandivt. Herpétologie de la Vienne. Poitiers 1844 .

DuPRÉ. Tableau indicateur des principeaux monuments historiques des sites et des curiosités naturelles de Poitiers et de ses environs. Poitiers, Dupré, s. d., in $8^{\circ}$.

Batgier et $\mathrm{Ch}$. Arnauld. Monuments religieux, militaires et civils du Poitou. Niort, Robin, in $4^{0}$.

Pitre-Chevalikr. Les Noces vendéennes. Musée des familles 1845-1846 p. 207-208.

Détails sur la danses; chansons de noces.

De LA Liborlik̀re. Vieux souvenirs du Poitou d'avant 1789. Poitiers, 1846. (Notice sur la grand' Goule.)

FII.LoN (Benjamin). Recherches historiques et archéologiques sur Fontenay. Tome I. Fontenay, Nairière-Fontaine, 1846, un vol. in 80 br. (T. I, le seul paru.)

Il existe quelques exemplaires du sec. vol., tous incomplets.

Journal de Guillaume et de Michel Le Riche avocats du roi a St. Maixent (de 1534 a 1586) publ. par La Fontenelle de Vaudoré. St. Maixent, Reversé, 1846 .

A la suite Requête presentée par les hab. de St. M. a Moreau de Beazumont, lieutenant du Poitou pour obtenir les entrées (en patois) d'apie, un imprimé du temps (V. 1748).

Babinet. Mélusine, Geoffroy la grande dent, lég. poitevines. Paris, Techener, 1847 , broch. in 80 .

Superstitions populaires dans l'Almanach du bon agriculteur pour l'annéc 1848 . Niort, Robin.

C'est, dit M. Desaivre (Croy. etc. p. 5), la confession d'un ex-sorcier, qui raconte ses anciennes pratiques, tont en s'en moquant, $a$ un instituteur et ì ses ellèves.

Bussik̀re (Th. de). Histoire de Sainte Radegonde, reine. Paris, 1850, gr. in 80. Dans l'Introduction, récit populaire d'un miracle.

Giraudeau (J.). Précis historique du Poitou pour servir à l'histoire générale de cette province, suivi d'un aperçu statistique de la Vienne, des DeuxSèvres et de la Vendée. $S_{j} l_{\text {, }}$ in $8^{\circ}$, cartes. Cf. plus. art. de la Romania sur la Badia (de Niort) Couvent lupanar que l'on disait avoir été fondé a Niort par un comte du Poitou.

Longuemar (A. de). Chroniques et légendes populaires du Poitou, des Gaulois à l'an 1000, recueillies sur les bords de la Vienne, du Clain et de la Gartempe. Poitiers, $185 \mathrm{I}$, un vol. in $8^{\circ}$ avec carte et gravures.

Atiber. Recherches sur la Paroisse de St. Pierre Les Eglises, près Chauvigny-sur-Vienne. Mém. Soc. Ant. de l'Ouest Année 185I. P. 45-416: Usages et coutumes.

La Vendée en 1852 par le baron de Wismes. Gr. in fol. avec planches gravées. 
LUNrER (Dr.). Recherches sur quelques déformations du crâne observées dans le département des Deux-Sèvres. Mém. de la Soc. de statistique des Deux-Sèvres 1852.

Cette déformation n'était pas rare autrefois; elle était obtenue au moyen de l'armature d'une coiffure en carton posée sur la téte de l'enfant.

Mélusine poème relatif a cette fée poitevine comp. dans le $14^{e}$ siècle par Couldrette, publié pour la première fois par Francisque Michel. Niort, Robin, 1854 , in $8^{\circ}$.

Histoire de Mélusine princesse de Lusignan avec l'hist. de Geoffroy surnommé a la grande dent, par Nodot, précédé d'une introduction sur la légende de Mélusine. Niort, L. Favre, in $8^{\circ}$.

Duchejin. Récits du pays du Bocage, traditions, légendes et chroniques. Laval, 1855 , in $12^{\circ}$ de $400 \mathrm{p}$.

ThiaIs (David de). Le paysan tel qu'il est, tel qu'il devrait être. Actualité. Poitiers, Hileret, 1856 , un vol. in $8^{\circ}$.

Superstitions populaires dans l'Almanach du bon agriculteur pour l'année 1857 .

Les rives de la Vienne, légendes du Poitou, par le comte de Croy. Paris, 1857 , in $12^{\circ}$.

Marcel (T.). De la Bachelerie de Melle. Niort, Favre, 1857.

Détails d'anciennes maurs.

Ledain (B.). Histoire de la ville de Parthenay, de ses anciens seigneurs et de la Gatine du Poitou, portrait et carte. Paris et Poitiers, 1858, in 80.

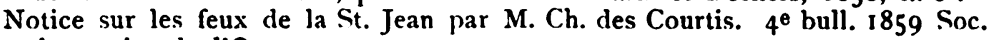
des antiq. de l'Ouest.

Bouchard (Henri Edm.). Annette Taudet ou les Sorciers du Poitou au XIXe siècle, croquis de mœurs d'après nature. Poitiers, Dupré, 1867 , in $12^{\circ}$.

Cf. sur ce livre la Revue de l'Aunis 25. Mai 1867.

Jozfau (Dr.). Elena, 1867.

Roman; détails sur les marais de la Sèvre.

Liborlikere (de la). La Grand' Gueule de Poitiers. Soc. des Antiquairs de l'Ouest 1867 , ler bulletin.

Chergé (Ch. de). Guide du voyageur à Poitiers.

Fir.ton (Benjamin). Lettres écrites de la Vendée à M. Analole de Montaiglon. Paris, Tross, 186 I (figures), in $8^{\circ}$. Tiré à 120 exemplaires, et non mis dans le commerce.

Superstitions dans Annuaire de la Soc. d'émulation de la Vendée 186I p. I42.

BÁlDRY (abbé). Antiquités celtiques de la Vendée et légendes. La Rochesur-Yon 1862, 1864, 1873. Extrait de l'Annuaire de la Société d'émulation de la Vendée 1872 p. $110-136$.

Airbarer. (abbé). Pèlerinage de N. D. de Pitié. Poitiers, Oudin, 1866.

Kondlek. Vie de Saint.Junien, Poitevin et bénédictin, patron des laboureurs du Poitou. Niort, Clouzot, 1866 , un vol. in $8^{\circ}$.

Raudry (abbé Ferlinand). Mémoires lus au Congrès archéologique de France. (Fontenay le (Comte 1864). Niort, Clouzot, 1865.

Callifet (Pierre). Jes Veillées du Mardigras, entretiens sur l'agricultur. Niort, Mercier, 1867 , in $18^{\circ}$.

CaIt.tet (Pierre). Michelle, Roman Poitevin, 1868.

Ces deux romans contienent des détails sur les maurs.

Nicolıìre (Stephane de la). Une paroisse poitevine. Nantes, 1866.

Vinum-Grandmarais. Tableau des Serpents de la Vendée. Nantes, 1868.

Gat.l.te (Ch. Edouard). La ville de Beauvoir sur mer (Vendée). Nantes, 1868 , in $8^{\circ}, 217 \mathrm{p}$.

Beauchet-Fit.teau. Simples notes sur quelques pèlerinages pieuses pratiques, usages etc. du diocèse de Poitiers. Paris, 1869 , in $8^{\circ}$.

I.onguemar (de). Géographie populaire du département de la Vienne. Poir tiers, Létang, 1869 , un vol. in $12^{\circ}$.

Buffíi:Res (I..). Petite géographie communale, histoire, biographie, statistique et usages locaux du département des Deux-Sèvres, avec I carte coloriée, etc. Niort, L. Favre impr. In $16^{\circ}, 96 \mathrm{p}$. 
Filion (B.) et O. De rochebrune. Poitou et Vendée. Etudes historiques et artistiques avec eaux-fortes par Rochebrune. In $4^{\mathbf{0}}$.

Desaivre (Léo). La chasse Gallery. Revue de la Saintonge, du Poitou et de l'A unis 25.0ct., 29 nov. I 869.

Drsaivke (Léo). Gargantua en Poitou avant Rabelais. Broch. in $8^{\circ}$. Extr. de la Revue de l'Aunis, de la Saintonge etc. du 25 juin I869 t. IX p. 354 è suiv. avec additions.

Lit̂vre (A. F.). Notes sur Couhé et ses environs. Niort, Clouzot, i 869, 2 vol. in $8^{\circ}$.

Détails sur Mélusine.

TrEsSAy (abbé de). Quelques mots sur l'Ile d'Yeu. Luçon, I869. Quelques traditions.

BEAUChet-Filleau. Pièces inédites rares ou cur. Concernant le Poitou et les Poitevins. I870, in $8^{\circ}$. (Rachât d'un repas dû par le commandeur d'Ensigué, appelé la tripe.)

Hugurs Imbert. Histoire de Thouars. Extr. des Mém. de la Soc. de stat. des Deux-Sèvres 1870 .

Clémentr-Prieur. La Vendée en 1873. Angoulême, Nadaud. In $8^{\circ}$.

Hugues Imbert. Une quenouille de mariage avec planche. Notice sur certains droits du Sgr. de Thouars. Bull. de la Soc. de stat. des Deux-Sèvres 1876 p. 78 .

Barbier de Montault. Jeu de la Quintaine en Poitou dans la Commune de Chasseneuil. Mém. de la Soc. des Antiq. de l'Ouest 1874, 145-146.

DESAIVRe (Léo). Le coq, la poule et l'œuf. Dans Bull. de la Soc. de stat. des Deux-Sèvres 1876 p. I1 2.

- Le Serpent, le Lézard et le Crapaud. Niort, Clouzot. Bull. de la Soc. de stat. des Deux-Sèvres 1877 p. 334 .

Usages et traditions du Poitou - Dans la préface de Lalanne: Glossaire du Patois Poitevin. Mém. de la Société des Antiq. de l'Ouest t. XXXII, année 1877 p. XIII--

Buche de Noël - Guilloneu (cf.p. 160) - Feu de St. Fean - Ensorcellements - Gâteaux de Fête. (Naulet, forme grotesque d'un petit en. fant - Merlusine).

LAISNEt, DE la SAJde. Croyances et légendes du centre de la France. 2 vol. in $8^{\circ}$ (plus. faits relatifs au Poitou).

Desaivre. Essai sur le Noyer et le Pommier. Niort, Clouzot, 1879, in $8^{0}$ de I9 p. Extr. des Bull. de la Soc. de stat. des Deux-Sèvres.

- Jeux et divertissement populaires en Poitou avant la Révolution. Niort, 1879, in $8^{\circ}$ de 24 p. Extr. des Bull. de la Soc. de stat. des Deux-Sèvres.

- A propos du saut de Verruyes. Bull. de la Soc. de statist. des DeuxSèvres 3.12. 1876 .

- Analyse d'un Mémoire relatif au préjugé superstitieux sur les Sorciers et les devins dans les départements de l'Ouest. Bull. de la Soc. de stat. des

- Deux-Sèvres 1880 p. 273 .

- Etudes de Mythologie locale: I. Les Abeilles; II. Noël; III. les Oiseaux. Niort, Clouzot, 1880, in 80 de 14 p. Extr. des Bull. de la Soc. de statist. des Deux-Sèvres.

Sovchí. Croyances présages et Superstitions diverses. Niort, Clouzot, 188I, in $8^{\circ}$ de 32 p. Extr. des Bull. de la Soc. de stat. des Deux-Sèvres.

DESAIVRE (L.). Croyances présages, usages, traditions diverses et proverbes. Niort, Clouzot, 1881, in $8^{\circ}$ de 39 p. Extr. des Bull. de la Soc. de statist. des Deux-Sèvres.

SovchÉ. Proverbes, traditions diverses, conjurations, formulettes et devinettes. Niort, Clouzot, I 881 ( 1882 ), in $8^{n}$ de 82 p. Extr. des Bull. de la Soc. de stat. des Deux-Sèvres.

Desalvre: (Léo). Formulettes et enfantines du Poitou. Niort, 1881, in 80 de $35 \mathrm{p}$.

BEAUCHET-FInleAu (H.). Croyances, superstitions, médecine, usages et préjugés du canton de Chef-Boutonne. Bull. de la Soc. de stat. des DeuxSèvres $188 \mathrm{I}-82$ p. $543-565$. 
Desalvk: (L.). Etudes de mythologie locale. Le monde fantastique. I 882, in $8^{0}$ de 25 p. Dans Bull. de la Soc. de stat. des Deux-Sevres.

Strbuloco (Paul). Gargantua dans les traditions populaires. Paris, Maisonneuve, 1883 , in $12^{\circ}$.

Le chapitre $V$ (de la page 170 ì la page 185$)$ est relatif à Gargantua en Poitou.

R a ces ma udites.

Ouvruges à consulter.

MICher (Fr.). Hist. des races maudites (Calots du Poitou).

ArNal:LI) (Ch.). Hist. de l'abbaye de Maillezais. Niort, Robin. p. 3. (Colliberts du Poitou).

Cfr. sur les Colliberts:

Alfren Richard. Mém. de la Soc. des Antiq. de l'Ouest t. XXXIX Dis. cours de la séance publique annuelle.

Arcíre (P.). Hist. de la Rochelle et du pays d'Aunis I p. 96.

Dufol'r. De l'anc. Poitou et de sa capitale p. I2I.

Savary. Notices sur les Huttiers de la Sèvre. Mém. de la Soc. de statist. des Deux-Sèvres 1838 - -39.

De ia Fontenilt.j: de Valmorí. Siatistique de la Vendée p. 93.

LatGardeli.E. Note anthropologique sur les Huttiers de la Sèvre, revue d'Aunis 1869 p. 343, etc. etc.

\section{Contes.}

Fur.LON (B.). Frère Fadet. Revue des provinces de l'Ouest t. I p. 243 sqq.

Pofy D'Avant (Mlle). La Mouété de quene (Moitié de Cane). Dans Revue des prov. de l'Ouest 1858 . Tirage à part. Nantes, 1859, in $8^{\circ}$.

VAN DFr Cruyssen. La messe nocturne. Soc. de statist. des Deux-Sevres, Bull. $\mathrm{I}-3,1880$.

Desaivre (Léo). Le curé de Parthenay le vieux, conte analogue au précedent id.

Dovar.t. (Charles). Poésies. Paris, Charpentier, 1868 , in $18^{\circ}$. (I.a chaşse invisible. Conte en vers.)

Les gens de Saint-Maixont sont de la part de leurs voisins l'objet de facéties et les héros d'histoires drôles, un numéro de l'Ancien Figaro contient sur ce sujet un article de M. de Coralles. - M. Gelin, ancien instituteur, a recueilli beaucoup de contes, de traditions et de légendes qu'il se propose de publier.

C, hansons et danses.

Frokkrs: Ouvr. cité, p. 462 . Chanson de la mariée. Dellx versions en français.

Vitr.ecirr.f. (de la). Ouvr: cité. Chanson de la mariée en patois. Citće clans Instr. rel. aux poésies pop. com. figurant à la p. 26 de Notice arch. sur Chavague en Paillers.

Instructions relatives aux poésies populaires de la France. Impr. imp. 1853, in $8^{0}$. p. 49. Rossignolet des bois, rossignolet sauvage, recueillie pres de Niort par M. de Corcelle.

p. 50. Le Rossignolet des bois.

Massí (Isidore). La Vendée poëtique et pittoresque. Cont. chans.

Bijeadu (Jérôme). Chants et Chansons populaires des provinces de l'Ouest, Poitou, Saintonge, Aunis et Angoumois, avec les airs originaux recueillis et annotés. Niort, Clouzot. 2 vol. gr. in $8^{\circ}$. Extr. des Mém. de la Soc. de stat. des Deux-Sèvres.

La Villemarqué (H. de). Analyse des chants et chansons populaires des provinces de l'Ouest. Bulletin du bouquiniste, I nov. 1866.

Bfaut.ıeU (Désiré Martin). Mém. sur quelques airs nationaux qui sont dans la tonalité grégorienne. Extr. des Mém. de la Soc. de stat. des Deux-Sèvres.

Chansons Vendéennes avec la traduction. Mémoires de l'Acad. Celtique t.III p. $370-383$. Musique à la fin du volume.

Zeitschr. f. rom. Phil. VII. 
Röbin. Etude sur certains airs de danse du Poitou au XVIe siècle. Bull. de la Soc. des Antiq. de l'Ouest, $9^{\mathbf{e}}$ série.

Cf. G. Bertrand, Rev. des Soc. Sav. 4. sér. t. III p. 283.

De la Marsonniere. Coup d'oeil sur la poésie poitevine. Bull. de la Soc. des Antiq. de l'Ouest 1860, $2^{\circ}$ Bull.

- Etude sur la gente poitevin'rie. Mém. de la Soc. des Antiq. de l'Ouest 1858-59. - Voir plus loin: Chansons en Dialecte Poitevin.

Founloux (Jacques du). La vénerie etc., précédé de quelques notes biogr. et d'une notice bibl. par Pressac, avec grav. sur bois. Angers, Ch. I.ebossé, 1844, gr. in $8^{\circ}$. Plus. airs poitevins notés.

Chanson poitevine: Al entrade del tens clar, citée en partie dans La Rousse. verbo. Vadurié t. XV p. 720.

Chansons poitevines publiées dans le Canard potevin. Journal hebd. en Patois Poitevin publié à Melle, Deux-Sèvres, in $8^{\circ}$.

Annonce du 20 août 1877 du Journal la Mélusine, Paris, Viaut.

BRANLes DU PoITot. Les Poitevins jouissaient au Moyen âge d'une grande reputation comme danseurs. Cf. le recueil de proverbes dit de l'A postoile. Paris, Crapelet, in $4^{0}$ et Abel Jouan, Recueil et discours du voyage du roi Charles IX (passage de la cour à Thouars). V. La description du branle dans l'orchésographie de Thoinot Arbeau. Les autres danses du Poitou sont descrites dans les Chants et Chansons populaires des prov. de l'Ouest de Jérôme Bujeaud. St. Maixent, 1866. Extrait des Mém. de la Soc. de stat. des Deux-Sèvres.

Gauthier (E.). Etudes sur les chants populaires de la Bretagne et du Poitou, recueillies et annotés par M. A. Guéraud. Nantes, 1859 .

Le travail de M. Armand Guírand, couronné en 1858 par la Société académique de Nantes, allait enfin paraitre quand la mort est venue surprendre l'auteur. Le ms. fut déposé à la bibl. de la ville de Nantes. (Note des Chants et Chansons populaires des prov. de l'Ouest de feu F. Bugeaud, bibliographie.)

Noëls.

Les Grands Noëls nouveaux en françois, en poitevin et en ecossois. Paris, in $8^{\circ}$, goth. s. d.

Il y a eu deix editions, cf. Nouvelles recherches t. II p. 107 et F. Denis. Revue de Paris t. XLVII p. 195-206, un article sur les Noëls, oì il donne quelques citations, mais françaises, de ce volume. (G. B.)

Recueil des plus beaux Noëls Poitiers 1668. Grande bible des Noëls. Plas. éditions de ces Noëls ont été imprimées à Orléans, à Poitiers et à Nantes pendant le XVIIIe siècle. (Glossaire L. Favre).

Gusteau (abbe). Noëls très-nouveaux dans tous les styles, par un pasteur a l'usage de sa paroisse. Fontenay, J. Poirier, 1738,48 p. in $12^{\circ}$.

Un avertissement dit: quelques uns de ces Noëls quoyque imprimez ailleurs, sont du même autheur. Cette impression est restée inconnue. (G. $B$,)

- Noëls très-nouveaux composé par un pasteur. Fontenay, 1738, nouvelle edit. en $1742,84 \mathrm{p}$. in $12^{\circ}$.

Contient p. 10, 23, 27 et 30 quatre Nö̈ls en patois; un fragment a sté reproduit par G. Brunet, Recueil de plusieur's pieces etc. p. $137-138$.

Gisteau (abbé). Noëls nouveaux dans tous les styles pour les différents goats par un pasteur, edition revue par l'autcur, augm. de notes curieuses

et d'une pastorale en cantique pour servir de rejouissance aux familles chrestiennes. Fontenay, Jacques Poirier, 1742 , in $12^{\circ}$. Avertissement, grav. sur bois (Armes de Fontenay), 84 p. (Favre Gloss. abbé Gusteau v. ci-après et ci-dessus.)

- Autre édition des Noëls de M. Fr. Gusteau $175^{6}$ avec avertissement in $12^{\circ}$, $120 \mathrm{p}$. Fontenay, Chambonneau imp. du roi. (G. Bardy, préface des poésies lè l'abbé Gusteau v. ci-après.)

- Plúsieurs autres éditions: en 1776 avec avertiss. nouv. bois représentant David jouant de la harpe, en 1789 édition différente contenant plus de $120 \mathrm{p}$. etc. etc. 
- Poésies patoises ornées d'un portrait de l'auteur avec glossaire par Pressac sousbibliothécaire de la ville de Poitiers et notices sur l'abbé Gusteau et Pressac. Poitiers, H. Oudin, $1855-6 \mathrm{I}$, et sur la couverture Niort, Mde Clouzot et fils, I 862 .

Contient plusieurs Nö̈ls et Chansons dont une pour la cérémonie du gâteau et du bouquet de la marice qu'on chantait encore dans ma jeunesse dans les villagres. - En attendant les adieux de ses compagnes, il itait traditionnel que lu mariée fondit en larmes. - Compliment sous forme de dialogue ì l'évîque de in Rochelle (Roch de Menou), que j"ai entend" encore réciter. La misire daux paysans au sujet daux mangeoux. Première églogue de Virgile rappelant une traduction faite par un autre patoisier poitezin, le curé Babu.

Noëls poitevins (au nombre de 87 ), manuscrit indiqué au Bulletin du Bibliophile publié par la libr. Techener, 2e série $n^{0} 37$.

Nouveau recueil des plus heaux Noëls (français et poitevins). Poitiers, Oudin. s. $d$. in $12^{\circ}$.

- Recueil de Noëls. Poitiers 1824; quelques pièces en patois.

- Nouveau recueil des plus beaux noëls poitevins. Niort, Robin, I845, un vol. in $12^{\circ}$.

LE Motcise. S'en suivent plusieurs chansons de noëls. Nouelz nouveaulx. Paris, 1520, pet. in 80 , goth., $63 \mathrm{fts}$.

L'auteur étnit curé de Saint-Georges du Puy-la-Garde, en Poitou. Ce volume, oì se montre une grande naïveté, est d'une rareté extrême; un exemplaire fait partie ds la belle bibliothèque de M. Cigongne, achetén en bloc par le duc d'Aumale (catalogue, no 1284). La Société des bibliophiles français en a donné une édition nouvelle (Paris, Lahure, 1860, in 160, XVI et 172 p.); elle n'a ité tirée qu'à 36 exemplaires; on $y a$ joint les noëls composés (vers 1524), par les prisonniers de la Concier. gerie et deux autres tirie du Recueil des noils du plat d'argent. (Poly. biblion.)

Danık. (Jean). Dit maître Mitou: Noëls (1520-1530), précédés d'une étude sur sa vie et ses poésies par Henri Chardon. Le Mans, Monnoyer, 1874, un vol. in $8^{\circ}$. (Noëls en patois poitevin du XVIe siècle.)

Recueil des plus beaux Noëls, choisis entre tous ceux qui ont paru jusqu'd présent. Poitiers, Barbier, 1824, un vol. in $12^{\circ}$.

Nouveau recueil des plus beaux noëls. Poitiers, Barbier, 1838, in 12:

Cantiques spirituels composés par Messire J,ouis Marie Grignon de Monfort prêtre et missionairc apostolique, décídé en odeur de Sainteté à St. Laurent sur Saivre (sic) le 29 avril 1716. $4^{0}$ idition. Niort, Jacob Desbordes, 1749. in $12^{\circ}$.

Cantiques des missions par L. M. Grignon de Monfort. Poitiers, 1779, in $12^{\circ}$.

Cantiques des missions comp. par L. M. Grignon de Monfort, prêtre et mission. apost., né en Bretagne le 31 Janv. 1673 et $\mathrm{m}$. en odeur de sainteté en fais. la mission à St. Laurent sur Sèvre en Poitou le 28 Avril 1716. Poitiers, Fr. ainé Barbier, 1817 , in $12^{\circ}$, avec bois. (Ces cantiques sont encore trèsrépandus et constituent un livre populaire si non patois.)

Charon (Louis Pierre, paysan Vendéen). Précis historique de la prétendue église-française, dans les communes de Pouillé et de Petosse (Vendée) de: diée aux hab. des. Campagnes. Fontenay, Nairière Fontaine, 1843.

\section{Devine tites.}

Drsaivre (L.). Formulettes et enfantines du Poitou. Niort, Clouzot, 188I, in $8^{\circ}$ de 35 p. Extr. des Bull. de la Soc. des statist. des Deux-Sèvres. Contient 39 devinettes.

Sor:che. Proverbes etc. ouv. cit. Contient 66 devinettes.

Desajvre (L.). Journal la Mélusine p. 246. 
Formulettes.

Dgiarvre (L.). Ouvr. cité et Mélusine p. 366.

Souché. Proverbes etc. ouvr. cit.

Dom Chamard (bénédictin de Ligugé). Bull. de la Soc. des Antiq. de l'Onest, $4^{e}$ trimestre 1865 . Note relative à une formule d'incantation.

Desarvre (Léo). Prières populaires du Poitou. Bull. de la Soc. de statist. des Deux-Sèvres 1883.

\section{Proverbes.}

CaIllet (Pierre). Les Veillées du Mardigras, ouvr. cité.

Desalvre (L.). Croyances etc. ouv. cité.

Contient a la fin des prozerbes.

SouchE. Croyances (passim). Proverbes, Traditions diverses (etc.).

Contient 120 proverbes sur les Mammifires et les Oiseaux. II y' en a.

d'autres dans le même outrage.

\section{Costumes.}

Monball (comte E. de). Monuments religieux, militaires et civils du Poitou. (Département de la Vendée.) (Coiffures des femmes de Fontenay, Les Sábles d'Olonne, Bourbon-Vendée etc.)

Vues et Costumes pittoresques du département des Deux-Sèvres par P. Gellé avec texte par $\mathrm{Ch}$. Arnaud. Niort, typo.-litho. Morisset. Contient seulement 4 lith.: Sortie de l'Eglise St. André de Niort, les Grisettes de Niort, une foire à Niort, un marché à Niort; resté incomplet.

Il existe plusieurs autres lith. de Gelle représentant des scènes villageoises vendues séparément et dant il est tris-difficile de réunir aujourd'hui une collection complète. L'une représente le philanthrope Sauquet Favelot distribuant des aliments aux pauvres, titre: Sauquet favelot; une autre une noce villageoise; une $3^{\mathrm{e}} u n e$ noce (a Niort); une $4^{\mathrm{e}}$ une assemblée de village; une $5^{\mathrm{\theta}}$ en deux tableaux, la mariage à l'jglise, le bouquet de la mariée; une 60 une villageoise allaitant son enfant et une scine d'abreujoir (aussi en deux tableaux); une $7^{\mathrm{e}}$ la veillée de Noël dans une ferme du Poitou; une 8e ladoration des bergers du Poitou: une ge des mendiants etc. etc. - P. Gellé a fourni des dessins aux auvres agricoles de Facques Bujault qui ont paru par fragments dans lalmanach de Morisset et ont été réunies en un vol. sous le titre: Ciuvres de Facques Bujault, illustrées de 34 sujets graz'és sur bois par Guillaumot d'apris les dessins de Gelle. Il est aussi l'auteur de la lithographie du premier. volume de Thistoire de ladministration supérieure du departement des Deux-Stures representant la proclamation de la patrie en danger dans un village des Deux-Sizires et de diverses itudes lithographiées. -

Suite de Costumes nos I i 6 . Lith. de Charpentier. Charpentier père et fils et Cle., Editeurs à Nantes. - Département des Deux-Sèvres.

I. Grisettes de Niort; 2. Laitiëres et marchandes de légumes de Niort; 3. Femmes des environs de Niort. Costumes d'été; 4. Paysannes des environs de Niort allant au marche; 5. Femmes des environs de Niort en grande mante; 6. Paysannes des environs de Niort.

Niort in histoire des principales villes de France de L. Favre. Niort, Rohin, gr. in $8^{\circ} \mathrm{s}$. $\mathrm{d}$.

MÉRIMEE (P.). Lettres à une inconnue.

Il y est question de la coiffe des femmes de la Gâtine (Parthenay') qu'il assure remonter au $X V$ e siècle.

A l'occasion du concours régional de Niort, M. H. Gelin, ancien instituteur a publié dans le Mémorial des Deux-Sèvres 2 Mars, 18 Mars et 8 Juin 1882 des articles sur les costumes de la région.

Les institutrices avaient envoyé à l'exposition scolaire des poupées habillées suivant la mode actuelle, et en même temps plusieurs spécimens de modes anciennes. M. Gelin avait émis plusieurs idées originales et formé des groupes de costumes. 


\section{Livres populaires, almanachs.}

Histoire de la vie, grandes voleries et subtilités de Guillery ct de ses compagnons; et de leur fin lamentable et malheureuse. Paris, 1608, pet. in $f^{n}$. Réimprimée a Troyes et ailleurs et dans les Hist. tragiques de $\mathbf{F}$. Rosset, rèimpression récente $(1848)$ par $\mathrm{B}$. Fillon a Fontenay chez Robuchon sous ce titre: Histoire veridique des grandes et exécrables voleries et subtilités de Guillery depuis sa naissance jusqu'à la juste punition et de ses crimes, remise de nouveau en lunière. In $3^{0}$.

- La prinse et deffaicte du capitaine Guillery qui a esté pris avec 62 voleurs de ses compagnons qui ont esté rouez en la ville de La Rochelle le $25^{c} \mathrm{dc}$ Nov. 1608; avec la complainte qu'il a faicte avant de mourir. Paris jouxte la copie imprimée ì La Rochelle par les héritiers de Jerosme Hautain I609, pet. in $4^{0}$.

Reproches du capitaine. Guillery faits aux carabins, picoreurs et pillards de larmée des princes. Paris, de l'impr. d'Ant. du Breuil, 1615, in $8^{\circ}$.

La complainte de (iuillery souvent publiée (en patois poitevin) se chante encore aujourd'hui.

Collet le célèbre voleur, mort au bagne de Rochefort vers 1835 , a aussi joui l'une grande célèbrité en Poiton. Ch. Moreau imprimeur à Melle a publić les: Mémoires d'un condamné ou Vie de Collet.

Les autres publications populaires locales n'ont guère plus d'importance et ne meritent point une mention spéciale.

Dr. Chabot Conseils aux cultivateurs sur leur santé ou précis d'un cours d'hygiène fait à l'école primaire de St. Romans les Melle. Melle, Moreau, $184 \mathrm{I}$, fort. vol. in $18^{\circ}$.

La ministresse Nicole. Dialogue Poitevin de Josué et de Jacob. Poitiers, H. Oudin, s. d. l'original serait à la bibl. nat. d'après le gloss. de Favre.

Jean Babu curé de Soudan. Eglogues Poitevines sur différentes matières de controverse. Nyort, J. Elie, 1701. Réedité par L. Favre. Niort, 1875.

Du même. Les deloicremont d'in oncien des Huguenots de Chondoné apré la rouine do préche, sur tout ce qui s'est fait et passé pendant la démo. lition du temple le treicième septembre i663. Poitiers, Pierre Amassard, s. d. $8 \mathrm{p}$. in $8^{\circ}$, attribuée ì tort à Drouhet.

L. Favre Gloss. cite deux éditions; réedité dans son glossaire. babu avait iélèbré aussi la destruction des temples de St. Maixent et de la Mothe. (ifr. la redition de la Moirie et autres auvres poétigues de fean Drouhet par Alfred Richard 18-8, Notice sur F. Drouhet p. II.

Fean Babu avait peut-ître emprunté son titre à faques Béreau Poitevin. "nuteur de's sglogues et autres a:uz'ves poitiques, a Poitiers, par Bertrand .Vosiereau, maistre imprimeur de la dite ville $M D L X V$. La $3^{c}$ egloguc ie Béreau a sté réimprimée par lieversé à St. Maixent, 1869, gr. in 8n, tirc a 25 exemplaires avec courte notice de M. Charles Dugast-Mutifeux. La Bibliographie poitevine de $M$. Clunzot (1878) indique une réimpression sous forme de placard a 10 exempl. s. l. n. $d$.

L'effondrement du palais de Justice de Fontenay le Comte arrivé le 8 Janv. 1699 suiri d'un poëme sur le $\mathrm{m}$. sujet et de stances à la gloire de $M$. le maire perpétuel de cette ville. Poüme burlesque en $3 \mathrm{p}$. dont une en patois poitevin du XVIIe s., attribué à Duchesne de Denant, publ. par les soins de B. Fillon. Niort, Clouzot, 1866 , broch. in $8^{\circ}$.

Dialogue de la mère Caquet et de Jeanneton par C. P. (Charles Palliot) en patois poitevin. Fontenay vers $1850,4 \mathrm{p}$. in $8^{\circ}$.

Le même auteur, aujourd'hui décédé, a publié plus. articles patois dans l'Indicateur de Fontenay.

Le fournal le Mellois s'est surtout fait remarquer par plus. publications de ce genre; divers pseudonymes cachent les noms de M. M. Moussaut d. m. p. Rondier, Beauchet Filleau eti. Des lettres patoises ont aussi paru dans ces derniers temps dans le Mémorial des Deux-Sèvres et la Revue de l'Ouest de Niort. Nous croyons devnir signaler plus spécinlement celles qui sont signées Feandu. 
Lanterne magique comique, historique, politique, morale et locale, oi l'on verra journal, animal sans égal et autres, le tout à l'occasion du carnaval. Prix 75 centimes au profit des Polonais. Niort, Morisset, $183 \mathrm{r}$, in $8^{\circ}$.

Grand catéchisme national par André Bonin, de St. Gelais prés Niort (Deux-Sèvres). Niort, Dépierris (après 1830).

Jardin d'amour ou Catéchisme des grandes filles, très répandu dans ma jeunesse et que je ne puis retrouver, in $12^{0}$ ? n'était point immoral mais stupide. Les amants bien éduqués se récitaient le jardin d'amour d'un bout a l'autre - c'est un dialogue.

\section{A l m a n a ch s.}

Vienne.

Le Poitou est un pays d'almanachs. Cette série se présente dès le régne de Louis XIII sous forme de calendriers de cabinct ou placards. Après eux, L'Almanach de d'Argoly romain et Poitevin grand astrologge (sic) cst signalé dès l'année 1673 , on le croit même antérieur à cette date.

- L'Almanach d'Argoly change un instant de nom à la fin du siècle dernier pour reprendre en l'an XII son ancien titre qu'il conserve jusqu'en I 833 .

Il existe des almanachs imprimés à Niort et à Fontenay au type de d'Argoly.

- Le véritable almanach de Poitiers pour 1791 par Argoly romain grand astrologue. Niort, Jcan Beaujeau $\mathbf{M}^{\mathbf{d}}$ rue du chaudronnier près l'hótel des Cavaliers de la Maréchaussée.

- Le véritable almanach de Poitiers pour 1792 par Argoly romain grand astrologue. Fontenay, Chambonneau.

Quoique Talmanach d'Argoly de Poitiers füt tris-répandu, la collection en est dépourvue d'intérêt.

L'Almanach du Poitou (in $12^{\circ}$ ) antérieur à 1732 est en grand progrès sur son devancier sans pouvoir cependant être comparé à l'almanach provincial qui le semplace en 1772 et continue avec une année d'interruption (1791) jusqu'en 1792 (en cette dernière année sous le nom d'Almanach du dép. de la Vienne).

L'Almanach provincial du Poitou, véritable annuaire de la province, est a ce titre très-recherché des travailleurs.

Son format pet. in $12^{\circ}$ le fait facilement distinguer des autres almanachs publies a cette epoque tant en Poituu que dans les provinces voisines.

Dans ces derniers temps, la plupart des imprimeurs de Poitiers ont eu leurs almanachs. Celui de Dépierris prenait en 1840 le nom d'Alınanach de la Vienne, celui de Pellet fils ainé, celui d'Almanach populaire de la Vienne (1853), l'Almanach de H. Oudin portait le titre d'Almanach de bon laboureur $(1851)$ et meme celui d'Alnıanach agricole et instructif de Niort en 1863; il est vrai de dire 'qu'en 1787 l'imprimeur Pierre Elies de Niort avait fait un almanach de Poitiers. Citons encore l'Almanach du bon cultivateur, Poitiers, A. Dupré, 1851: 52, et surtout le Calendrier Poitevin pour l'annće 1841 avec vue des principaux monuments de Poitiers. Poitiers, Pichot.

- Almanach de l'arrondissement de Loudun. Loudun, Mazeau, 1861.

\section{Deux-Sèvres.}

Nous n'avons point rencontré jusqu'ici d'almanachs Niortais antérieurs à Louis XVI. Les contrefaçons de l'almanach de Poitiers sont la preuve de la vogue dont jouissaient exclusivement les almanachs de cette ville.

On retrouve l'almanach de Beaujeau m"l rue du Chaudronnier sous le nom de Calendrier pour l'an VI. E. et P. Dépierris avaient peut-être importé ce nom, l'année précédente en imprimant un calendrier pour l'an V. Ce titre était conservé par eux en l'an VI. En 1793 Jean Joseph Dugrit faisait imprimér chez Le Franc Elies un almanach des Dcux-Sèvres.

La publication de l'Almanach de $P$. Elies, sans doute antérieure à 1787 , se continue sous la République et l'Empire, pendant plus. années, la petite brochure porte sur la converture un bois représentant Orion entouré des signes du Zodiaque, en 1816, il devient l'almanach Royal et passe en 1817 à Horisset successeur de P. Elies associé comme lui à Mile Vie Elies Orillat. 
En 1822 Robin succède à son tour à Mde Elies Orillat et l'association continue, en 1825 Morisset reste seul. En 1832 cet almanach prend un intérêt véritable par suite de la collaboration de Jacques Bujault qui donne successivement divers extraits de son agriculture populaire. En 1834, 35 et 36 cette publication produit, chose assez exceptionnelle, un supplément; l'almanach dès 1833 prenait le titre d'almanach du cultivateur et même de grand almanach du cultivateur à partie de l'annéc suivante. Les articles agricoles continuent après la mort de maître Jacques arrivée en 1843 et bientôt apparaissent les iliustrations d'un artiste local épris de la vie rurale, P. Gellé. Après être successivement passé aux mains de Mde Morisset (1851) et de ses gendres, cet almanach est aujourd'hui continué par M. Mercier (I 882). Il a donné deux fois (1845 et I86I) l'histoire surprenante et merveilleuse dc $M$. de $B$. (Boisragon) gentilhomme poitevin; puis L'histoire admirable et prodigieuse du jeune Creusé (1847) et enfin la sorcière et le gentilhomme (1848). L'Almanach de E. et P. Dépierris que nous avons signalé dès 1791 et qui porta un instant le nom de Calendrier, reprit sa dénomination d'Almanach sous la direction de la Vve Dépierris en l'an X; en 1811 , E. Dépierris ainé lui donnait le nom d'almanach de la préfecture, Cet almanach existait encore en 1837; a signalér comme annexe à l'almanach de 1809: Le Dialogue entre pierre *** de la commune de St. Liguaire et René *** de la commue de Bessine au sujet de l'entrevue de son maire avec S. M. l'empereur - en patois poitevin.

Robin que nous avons vu tout d'abord associé à Morisset fondait à son tour en 1832 l'almanach curieux des Deux-Sèvres, qui devenait en 1834 l'almanach des foires et marchés des Deux-Sèvres et en 1842 l'almanach du bon agriculteur titre que lui conservait en 1873 M. L. Favre, successeur de Robin. A signaler en 1839 l'histoire surprenant de la belle fée Mellusine, en 1840 l'hist. de Guy de Lusignan, roi de Jerusalem, et de son frère Urian, roí de Chypre, fils de Mellusine; en I84 I la vie de Geoffroi la grand' dent fils Mellusine et le combat entre un dragon ailé et un soldat Niortais; en $184_{2}$ la véritable chanson de la mariée (à partir de cette époque les deux variantes de cette chanson apparaissent tour à tour chaque année dans les divers almanachs Niortais); en 1846 Hist. extraordinaire et aventures merveilleuses de Louis Têtard, cultivateur vendéen. Cf. conte breton publié par E. Souvestre dans le foyer Breton. En 1848 et 1857 articles sụr les superstitions et préjugés populaires, etc. etc.

Almanach du père Gérard pour l'année 1792 la $3^{\circ}$ de l'Ere de la liberté par J. M. Collot d'Herbois. Niort, Lefranc Elies, 1792, in $12^{\circ}$.

Almanach des Muses de l'Ecole centrale du département des Deux-Sèvres. Niort, de l'impr. de E. Depierris, de l'an VI a l'an X. 5 tomes en 2 vol. in $12^{\circ}$, grav.

Almanach de l'association agricole du centre de l'Ouest pour les départ. de la Charente, de la Charente infírieure, des Deux-Sèvres, de la Vendée et de la Vienne. Niort, Rubin, 1846 , in $12^{\circ}$.

- Le méme Almanach pour 1847 . La Rochelle, Caillaud, in $12^{0}$.

Le double Almanach du cultivateur pour 1842. A Niort chez Bonneau près la barrière de Paris, Saintes Pathouot libr. (impr. à Limoges).

Almanach nouveau de Niort i831. Niort, Pathouot; Autres Almanachs des 30 dernières ann'ées: Almanach de Maitre Jacques, Almanach agricole, Almanach du bon laboureur, Almanach Napoléonien (1850), Almanach de la Saanté pour 1850 Niort, Morisset, pour 1851 Niort, Métivier. Calendrier d'horticulture florale par Despre\% ainc. Niort, Desprez, 1867.

Parthemay a publié un Almanach de la République Française en 1881.

\section{St. Maixent (Deux-Sèvres).}

L'Almanach Lainé porte différents noms, en l'an II Etrennes de la raison ou Calemdrier républicain: entretiens de l'écclectique Phocion avec ses frères de la Campagne. Lainé, St. M.; en l'an IV c'est le Calendrier républicain. Lainë, St. Maixent; en l'an V et 1797 Calendrier grégorien et républ. par d'Argoly, romain astrologue, F. Iainé, St. M. puis le simple titre de Calen- 
drier, ans VII. VIII. IX. XI et entin en 1807 , c'est encore le Calendrier grégorien par d'Argoly, romain astrologue. St. Maixent, François Lainé. En ces derniìres temps M. Ch. Revené a imprimé plusicurs des Almanachs qui ont remporté le prix fondé par facques Bujault pour la meilleure de ces utiles publications.

\section{Vendée. Fontenay.}

Les plus anciens almanachs de la Vendée paraissent être ceux de Fontenay. En l'an VII le citoyen Esnard defenseur officieux ì Fontenay le peuple publie chez Cochon, l'Almanach du départ. de la Vendée avec un précis historique sur quelques lieux principaux du départ. (Il y est parlé de Mélusine à propos de Vouvent). Nous avons signalé antérieurement (I792) un almanach au type d'Argoly che\% Chambonneau, impr. à Fontenay. En 1816 la Vie Beaujeau donnait l'Almanach nouveau. Habert (Aug. Vict.) eut ensuite ses annuaires (1826 etc.), vers le même temps (1826-36 ..) apparait l'Almanach royal de Petitot puis l'Almanach Vendéen de Gaudin fils (1838-44), qui donnait encore le double Almanach Vendéen en r846, La Vve Fillon, successeur de Gaudin, imposait à son tour le nom de grand almanach Vendéen à cette publication ... 1862-68 ...

L'Almanach national du départ. de la Vendée de Robuchon (1850) prenait plus tard vers I 868 la dénomination d'Almanach général de la Vendée.

Enfin Nairière-Fontaine confiait aux presses de Gaudin fils en 1843 les Etrennes Vendéennes, d'abord imprimées à Nantes (1840) et à Luçon (1841).

\section{La Roche sur Yon (Vendée).}

L'Almanach de la Roche sur Yon, sorte d'annuaire publié avec soin portait en 1809 le nom d'Etrennes de Napoléon et du départ. de la Vendée. En 1816. I7 etc. celui : d'Etrennes de Bourbon Vendée et du départ. plus tard Etrennes du départ. de la Vendée ou plus simplement encore Etrennes de la Vendée ... 1823. $1830 \ldots$

Luçon a aussi son almanach depuis quelques années, etc. etc.

Chansons en Dialecte Poitevin.

Chanson joyouse in lingage poetevinea fate do sege mis devant Poeters par l'Amiro. Poeters, 1569 , in 80 . Ch. B. réimpr. par de la Fouchardière l.c. La gente poetevin'rie, tout de nouvea racontrie ou Talbot ben et bea fat raiponse à Robinea. Avec le proces de Jorget et de son Vesin, ct chan. sons jeouses compousi en bea poictevin. Poeters amprimi tout avoure pre Emer Mesner 1572, pet. in fol. de $55 \mathrm{fl}$. sign. A-G iij lettres rondes fig. s. bois. (L. Favre gloss. Ch. B.)

I.a gente poetevin'rie etc. amprimi tout avoure a Poeters, pre la venfie Jon Blonchei demourant pre $\%$ le grond Horloge 1605 , in $8^{0}$ sans pag. (I.. Is. gloss.)

La gente poetevin'rie ouecque le precez de Jorget et de son Vesin et chansons jeouses compousie en bea poitevin et le precés criminel d'in Marcassin. Poeters, Jelian Thorea, 1620 , pet. in $12^{\circ}$. Ch. B.

La gente poetevin'rie etc. Poeters, Vie Jon Blonchet, 1620 , in $12^{\circ}$. (L. li.)

$\mathrm{La}$ gente poetevin'rie etc. Poitiers, Gabriel Garné, pet. in $8^{\circ} \mathrm{s} . \mathrm{d}$. Ch. B.

La gente poetevin'rie etc. Poeters, J. Flevrea, 1646 , pet. in $12^{\circ}$, arec une seconde partie intitulée Rolea divisi in beacot de pèces.

$\mathrm{La}$ gente poetevin'rie etc. recueillis et mis en ordre par J. Fleuriau. Avec le Rolea divisi. Poeters, J. Fleuriau, 1660 , in $12^{0}$.

$\mathrm{La}$ gente poetevin'rie etc. Poitiers $16 \%$ r. Ch. B. (Le meneloge de Robin $3^{\mathrm{e}}$ pièce de la gente poetevin'rie de 1572 par Jean Boiceau sgr. de la Borderie Jurisconsulte Poitevin) avait été imprimé pour la première fois à Poitiers à l'enseigne de la Fontaine en 1555 .

Rééditions récentes:

- La gente poitevinire (sic) aveque la procès de Jorget et de son Vesin et chansons ieouses compousi en bea poictevin, réimpression conforme à l'édition de 1572. Introduction par Alfred Morel-Fatio. Niort, Martineau et Nargot, 1877 , pet. in $4^{0}$. 
- Rolea divisi in beacot de peces ov l'universe ov poetevinea fat pre dialoge suivi de preces criminel d'in marcassin, réimpression conforme à l'édition de 1660 , pet. in $4^{\prime \prime}$. Niort, Martincau et Nargeot, 1879.

- La gente poetevin'rie ouecque le prece\% de Jorget et de San Vesin et chonsons jeouses compousie in bea poitevin. Av. introduction par L. Favre, pet. in $4^{0}$. Niort, L. Clouzot libr., 1878.

- Rolea divisi in bea cot de peces ov l'universeov poetevinea fat pre dialoge suivi du procez criminel d'in marcassin, pet. in $4^{n}$. Niort, L. Clouzot, 1878.

- Hymne, ode, couplets et ronde poitevine sur la naissance du roi de Rome par E. M. Dépierris jeune. Impr. à Niort 1811 , in $12^{\circ}$ de $16 \mathrm{p}$. (L. Favre glossaire.)

Fäiences, imagerie, plombs.

Finlon (B.). L'art de Terre che\% les Poitevins, suivi d'une Etudes sur l'ancienneté de la fabrication du verre en Poitou. Niort, Clouzot, 1864, in $4^{\prime \prime}$. Reproduit en partie clans Poitou et Vendée du même auteur.

Faïences d'Oiron (ouvrages à consulter):

BroNGNiART (A.). Traité des arts céramiques.

PUTTrer (A.). Monuments inédits pour servir à l'histoire des arts réưnis par Willemin (texte par A. Pottier).

SaIvetat (A.). Technologie céramique.

Labarte (Jules). Catalogue raisonné du Cabinet Debruge-Dumesnil (Introduction).

Borde (L. de la). Le chầteau de Boulogne.

ThORÉ et A. TÁnturier. Notice sur les faïences du XVIe siècle, ' dites de Henri II (Alliance des Arts I847).

L. Citemen' DF, Ris. Article in Gazelte des Beaux-arts 1860.

Derancie (père et fils). Recueil de toutcs les pièces connues jusqu'à ce jour de la fä̈ence française dite de Henri II.

De la Frerritre-Percy. Une fabrique de faïences à Lyon sous le règne de Henri II. Paris, Aug. Aubry, 1862.

Demmin (Auguste). Guide de l'amateur de faïences.

Prot (Eugène). Le Cabinet de l'amateur $1862 \mathrm{n}^{0} 19$, etc. etc.

Montargion (Anatole de). Les ceuvres de Maitre Bernard Salissy, réimprimées d'après les éditions originales etc. Fontenay le $\mathbf{c}^{\mathbf{e}}, \mathrm{P}$. Robuchou.

Enseignes de pélèrinage. (Ouvrages à consulter:)

RICHARI) (Alfred). Note sur quelques enseignes de pélèrinages. Bull. de la Soc. des antiq. de l'Ouest 1875 , I. trim.

Desalvre (Léo). Note sur une enseigne du Mont St. Michel au péril de la mer. Bull. de la Soc. de statist. des Deux-Sèvres 1881 , 10-12.

Tribert (abbé). Don d'une croix de pélèrinage du Notre Dame de Liesse. 20 Bull. de la Soc. des antiq. de l'Ouest 1882 .

La symbolique de l'armée Vendéenne. Cfr. Inventairc archéologique par Fortuné Parenteau, conservateur du Musée archéol. de Nantes. Nantes, Vincent Forest et Emile Grimaud, 1878 , in $4^{\circ}$.

Desaivre (Léo). Note sur un sceau présumé du Conseil supérieur de l'armée Vendéenne. Bull. de la Soc. de statist. des Deux-Sèvres 1881, $1-3$.

Mèreaux protestants. Cfr.:

LIÈVRe (Aug.). Histoire des protestants et des églises réformées du Poiton. 3 vol. in $8^{0}$. Paris, Grassart et Cherbuliez. T. 3 p. 362 et sqq.

Fil..on (B.). Etudes numismatiques.

Mall.aAd. Bull. du Protestantisme Fr. I p. 343, etc.

Imagerie populaire.

URBAIN GRANDIER. 1. Effigie de la condemnation à mort $\|$ au dessous bois représentant le curé de Loudun assis de face sur son bucher entre un bourreau muni d'une fourche qui attire le feu à droite et un magistrat à g. scène d'exorcisme dans une chapelle à g. derrière le magistrat, au dernier plan, l'église St. Pierre de Loudun $\|$ et exécution d'Uurbain Grandier curé de St. Pierre du Marché de Loudun, atteint et convaincu de magie, sortilèges et maléfices, lequel a esté brulé vif dans la dicte ville. \|F Fac-sim. très-réduit 
en tête du traité du célibat des prêtres par lêd. Urbain Grandier, curé de Loudun, opuscule inédit introduction et notes par Robert Luzarche frontispice à l'eau-forte de Ulm. Paris, René Pincebourde, 1866.

- 2. Pourtrait représentant au vif $\|$ l'exécution faicte à Loudun en la personne de Urbain Grandier || Prestre curé de S. Pierre et chanoine de l'église Ste. Croix dudit lieu atteint et convaincu des crimes de sacrilège, $\|$ magie sortilège, maléfice et possession: brulé tout vif par arrest des Juges conmissaires députez de par le roy $\|$ en la dite ville de Loudun, le vendredi 13 Aoust 1634. Exécuté le même jour. || Ensemble la copie de l'Arrest et les noms des ditz Commissaires.

Au dessous Urbain Grandier assis sur un bûcher, tourné à gauche, 3 bourreaux attisent le feu, prêttre arrosant le bûcher d'eau bénité à g., église à g., hérault à cheval à droite, diables dans la fumce etc. à droite et à g. de la composition se trouve un énoncé du procès.

A Poitiers par René Allain imprimeur et libraire demeurant en la rue notre dame la petite et sa boutique dans la grande allée du palais 1634 avec permission. In fol. reproduit grand. nat. en fac-simile in Gabriel Legué Urbain Grandier et les possedées de Loudun documents inédits de M. Charles Barbier. Paris, Ludovic Baschet, 1880.

Tout le reste de l'imagerie populaire poitevine - portraits de Ste. Radigonde, du père de Montfort (Grignon de) etc. appartient à l'hagiographie qui ne me parait mentionnée dans le travail.

Bois représentant Ste. Macrine semant dublé dans un sillon; Vo de 'la couverture inférieure de l'histoire abrégée de Ste. Macrine et de Ste. Pezenne près Niort par l'abbé P. P. (Picard) desservant de Magné. Niort, Morisset.

Théâtre populaire.

RABElaIs. Pantagruel Livre IV Chap. XIII raconte que sur ses vieux jours François Villon se retira à St. Maixent en Poitou sous la protection d'un abbé du monastère bénédictin et qu'il entreprit d'yfaire jouer la passion en gestes et languaige Poitevin. Il parle à ce sujet des diableries (mystères) joués au temps de Villon dans le parlouère de Poitiers (hôtel de ville?) et à Montmorillon. Plusieurs localités situées hors du Poitou sont aussi citées: Saumur, Angers, Doué, Langes (Langeais), St. Espain.

- Pant. Livre III Chap. XXVII, il est encore question de la Passion jouée a St. Maixent. Le parquet qui servait de théâtre etait sans doute celui de l'abbaye situé en face de la grande porte de l'église des bénédictins.

On a cru que la passion jouée à St. Maixent était le mystère de Jean Michel joué à Angers en 1486 à la lin d'août (v. J. B. Brunet, Manuel du libraire édition de 1862 t. III, 20 p. $1971-72$, verbo mystère).

Journal de Guillaume et de Michel Le Riche publié par de la Fọntenelle de Vaudoré. St. Maixent, Reversé.

Guillaume Le Riche qui avait lui mêne joué la l'assion à Poitiers le 19. Juillet 1534, où il y avait tant de peuple qu'il $\risingdotseq$ en demeura plus de la tièrce partie qui n'y entra - nous apprend que li 12 Juillet 1545 et autres jours précédents, 10 ou 12 jeunes gens commencèrent à jouer la Passion (à St.Maixent) ce qui dura 15 jours et $\mathfrak{a}$ la fin de chaque Mystère jouèrent une farce joyeuse.

Nous voyons enfin que le I Octobre 15,8 , des enfants de Paris jouèrent à St. Maixent et continuèrent à jouer des jeux en la sallc haute de l'aumónerie des femmes. Ils jouèrent du luth, des violes et chantèrent en musique en quatre parties (Journal de Michel le Riche).

Botchet (Jehan). Annales d'Aquitaine, Poitiers, Abr. Monnin, I644, p. 474 donne les détails suivants sur les mystères joués à Poitiers en 1534 (5 Juillet) "furent faites joyeuses et triomphantes monstres des mystères de l'Incarnation, nativité, Passion, ressurection et ascension de $\mathrm{N}$. S. Jésus Christ et de la mission du St. Esprit, lesquels mystère on joua quinze jours après au Marché Vieil de la dite ville, en un théâtre fait en rond fort triomphant et fut le dit jeu recommencié le Dimanche 19 dudit mois et dura onze jours continuels et subsécutifs où il y eut de très-bons joueurs, et richement 
accoustrés ... Ou joua aussi la Passion et Resurrection trois semaines après ou environ, en la ville de Saumur ou je vy d'excellentes feintes."

Le Journal de Généroux notaire à Partenay publié par M. B. Ledain dans les Mém. de la Soc. de statist. des Deux-Sèvres de 1862 donne les détails suivants sur les représentations scéniques de Partenay.

„Lc Jeudi 10 de Juin ( 1571$)$, jour de Trinité, je fis jouer áu carrefour de la Crolx du Marchioux de Partenay, la tragédie ou histoire d'Abel tué par Caïn, son frère." Le chroniqueur a voulu conserver le souvenir des acteurs, les rôles de femmes étaient tenus par des hommes, un festin suivait la représentation. L'année suivante à la même fête et au même lieu, Généroux fit jouer la tragédie de Médée ${ }^{1}$, dont il donne encore la distribution qui réveille la méme observation quand à l'absence des femmes.

„C'était, dit il, chose fort magnifique tant pour être bien jouée oue pour les feux artificiels et autres singularités.... La farce fut composée par Me François du Vignault $\mathrm{Sr}$ de Magot, de trois femmes qui trompèrent leurs marisjurés, l'un desquels je jouais.".

Nous ignorons si la tragédie patoise suivante a été jouée en son temps: Les amours de Colas comédie Loudunaise en beau langage, dédiée à $M$. M. les économes da,la Tour-volu (par St. Long). Loudun, R. Billault, 1732, in $8^{\circ}$. $\mathrm{Ch}$. B. cite une première édition.

Les amours de Colas, comédie Loudunaise en beau langage, dédiée à M. M. les oeconomes de la Tour-volu par S. L. Loudun, Chachereau, in $8^{\circ}$ de $2 \mathrm{ff}$. et $37 \mathrm{p}$. réédition chez Techener à Paris par les soins de M. Ch. Brunet en 1842 , aussi in $8^{\circ}$.

A la Révolution les tragédies reparaissent dans les campagnes, Dupin dans l'une de ses statistiques raconte que la mort de César fut représentée dans une grange à Champdeniers, bourg d'un millier d'âmes, les acteurs semblent avoir appartenu d la localité.

Le paysan écrivain, comédie en cinq actes par L. P. Charon, paysan vendéen, avec glossaire. Fontenay le cte, Gaudin père, vers 1840 , in 80 de $48 \mathrm{p}$. s. d. Gloss. Favre.

Atr.exiain (P.). Les élèves en chirurgic ou l'amour de l'hôpital, Vaudeville en un acte, représenté sur le théátre de Poitiers le 3 Janv. 1840. Poitiers, Dépierris, $184^{\circ}$, in $8^{\circ}$.

Le siège de Poitiers, drame lyrique en 3 actes et en vers, in $8^{\circ}$, indiqué dans le cat. Saurin frères, Poitiers, vers 1830 . patolster.

I par Jean de la Pérusse poitevin ami de Jean Boicean Jurisconsulte poiturlu ot

H. Gaidoz et Paul Stêbillor. 